\title{
New Thermoelectric Sensor Adapted to Realize an Infrared Radiations Detector
}

\author{
Mohammed Rahmoun, Khalid Hachami, Abdelwahad Touil, Benaissa Bellach, \\ Mohammed Bailich, and Ammar Merdani \\ Electronics and Telecommunications Team, School of Applied Sciences Engineering, University Med Premier, Oujda, Morocco \\ Correspondence should be addressed to Mohammed Rahmoun, moharahmoun@hotmail.fr
}

Received 7 April 2011; Revised 4 June 2011; Accepted 8 June 2011

Academic Editor: Jiun Wei Horng

Copyright (c) 2011 Mohammed Rahmoun et al. This is an open access article distributed under the Creative Commons Attribution License, which permits unrestricted use, distribution, and reproduction in any medium, provided the original work is properly cited.

The present paper deals with the design and the realization of a new thermoelectric sensor (Seebeck), sensitive to the infrared radiations emitted by different sources. The function mechanism utilizes radiative absorption phenomena, heat transfer, and thermoelectric effects. The sensor includes two printed circuits, the first is a planar thermoelectric circuit constituted of many plated differential thermocouples, and the second is constituted of a resistive constantan track and placed at the top of the first circuit so that the constantan track is placed on the top of the even thermocouple junctions. The constantan track, covered with a resin of great absorptivity, collects the infra-red radiations and generates temperature gradients between the junction points of the first circuit. Then the resulting temperature differences between the junction points are directly converted into a proportional Seebeck voltage. As an application, the sensor is adapted in order to realize a device for detecting infrared radiations, and the results are very encouraging.

\section{Introduction}

Infrared radiation is the electromagnetic waves in the wavelength region longer than the visible light wavelengths, lying from $0.75 \mu \mathrm{m}$ to $1000 \mu \mathrm{m}$. The wavelength region of $0.75 \mu \mathrm{m}$ to $3 \mu \mathrm{m}$ is often called the near infrared, the wavelength region of $3 \mu \mathrm{m}$ to $6 \mu \mathrm{m}$ the middle infrared, and the wavelength region of $6 \mu \mathrm{m}$ to $15 \mu \mathrm{m}$ the far infrared.

All objects with an absolute temperature of over $0 \mathrm{~K}$ emit infrared radiation. Infrared energy is determined by the temperature and surface condition of an object. Suppose there is an object that absorbs all radiant energy and appears completely black at all wavelengths. This object is called "blackbody".

The hot wire anemometer has been used for many years as a research tool in fluid mechanics [1]. The Seebeck effect was implemented for long time to conceive and realize a great diversity of sensors; the measurement of physical size is the difference in temperature between two points of a thermocouple [2]. The problems arising from the use of the traditional thermoelectric circuits are to solve the practical problems posed by the welding of many bimetallic contacts. The fabricating method of bimetallic circuits consists of deposit by electrolytic way a metal layer of high conductivity on a metal support of low conductivity [3-7]. In previous works $[8,9]$, we described and characterized a new thermoelectric sensor in a shape of printed circuit, adapted to differential circuit breaker and a fluid level detector. The present work is an experimental study of the coupling between a constantan track circuit used as heat capacitor and the bimetallic circuit. The two circuits are placed on the top of the other thus constituting a new thermoelectric sensor. As an application, the sensor is adapted in order to realize an infrared detector.

\section{Description}

The sensor includes two printed circuits maintained interdependent on Kapton support of $25 \mu \mathrm{m}$ thickness realized according to the famous engraving technique largely used in the printed circuit technology. The first circu,it as shown in Figure 1, is a constantan track with a thickness of $25 \mu \mathrm{m}$ and width of $0.4 \mu \mathrm{m}$. The second circuit as shown in Figure 2 is a thermoelectric track comprising a continuous ribbon of constantan of thickness $25 \mu \mathrm{m}$ available on Kapton of 


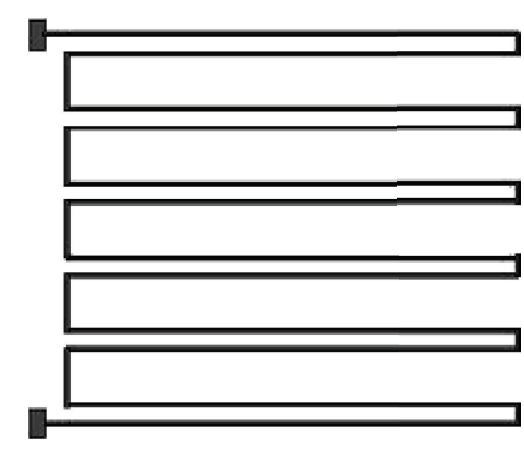

FIGURE 1: the constantan resistive track.

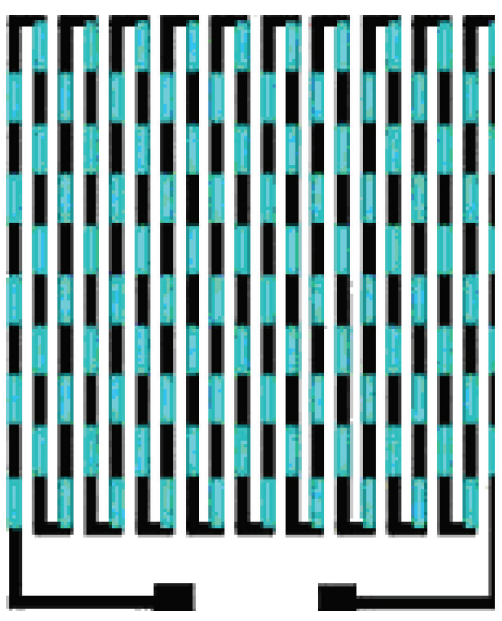

FIgURE 2: the bimetallic circuit.

thickness $25 \mu \mathrm{m}$. The ribbon is covered uniformly with an electrolytic copper deposit of high conductivity of thickness $5 \mu \mathrm{m}$. Using the photoengraving techniques, the circuit is engraved with iron perchloride following the form and the dimension desired. The ammonium peroxodisulfate attacks mainly copper and leaves intact the constantan sheet, is used to reveal the shape of the electrodes as shown in Figure 3 and the final form of the bimetallic circuit, shown in Figure 2, and is comprised of many plated differential thermocouples interconnected in series. The expression of the output voltage delivered by a thermoelectric chain (Figure 4) is only simple in the classic configurations where a great number of conductors of different nature are interconnected in series. Given that a bimetallic chain with plated electrodes is not made with homogenous conductors, it is necessary to determine the relationship between voltage and temperature differences by going back to the classical theory of thermoelectricity. In order to determine the expression of voltage delivered by Seebeck effect, it is necessary to determine the equivalent Seebeck coefficient expression of a bimetallic circuit.

Using the generalized Ohm's law that links the current density to the electric field and temperature gradient, the equivalent Seebeck coefficient $\alpha_{\text {eq }}$ in the copper plated regions [10] is given by

$$
\alpha_{\mathrm{eq}}=\frac{\alpha_{1} \sigma_{1} e_{1}+\alpha_{2} \sigma_{2} e_{2}}{\sigma_{1} e_{1}+\sigma_{2} e_{2}} .
$$
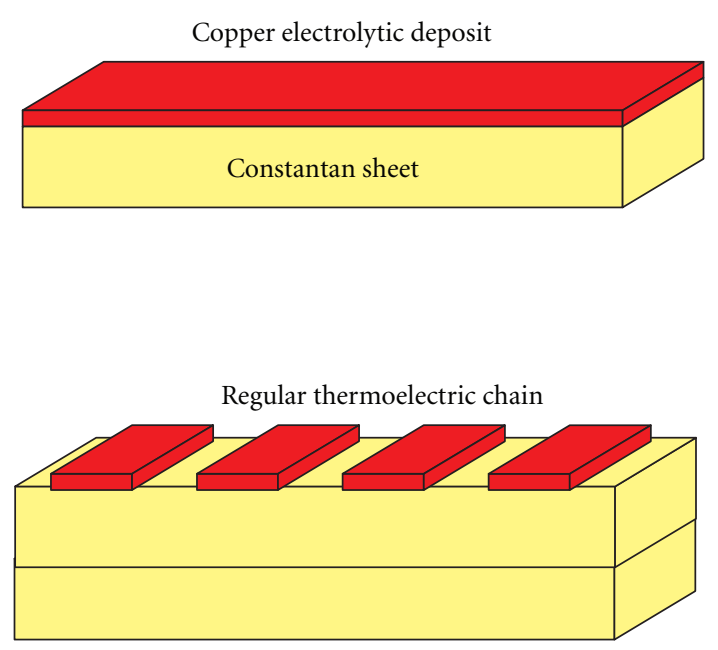

FIgURE 3: thermoelectric chain.
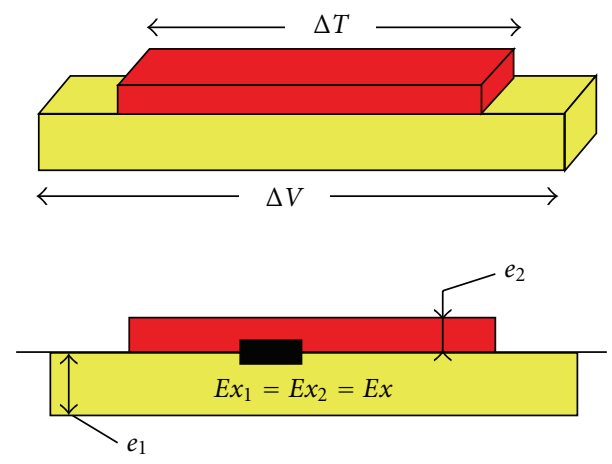

Figure 4: thermoelectric element.

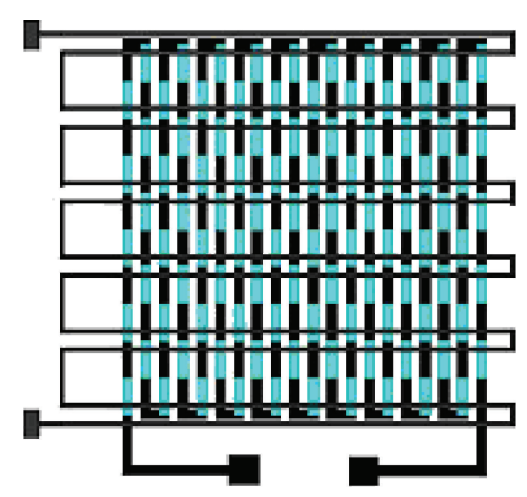

Figure 5: used sensor.

$\alpha_{1}, \sigma_{1}$, and $e_{1}$ are, respectively, the Seebeck coefficient, the electrical conductivity, and the thickness of constantan. The corresponding parameters for copper, are $\alpha_{2}, \sigma_{2}$, and $e_{2}$.

The equivalent Seebeck coefficient $\alpha_{\mathrm{eq}}$ approaches the Seebeck coefficient of copper and the temperature difference between two junction points is directly converted into a proportional Seebeck voltage: $\Delta V=\left(\alpha_{1}-\alpha_{\mathrm{eq}}\right) \Delta T$.

In order to realize a thermoelectric sensor, the first circuit (Figure 1) is placed on the top of the second one (Figure 2) so that the constantan track is placed on the top of the even junctions as shown in Figure 5. When the constantan 


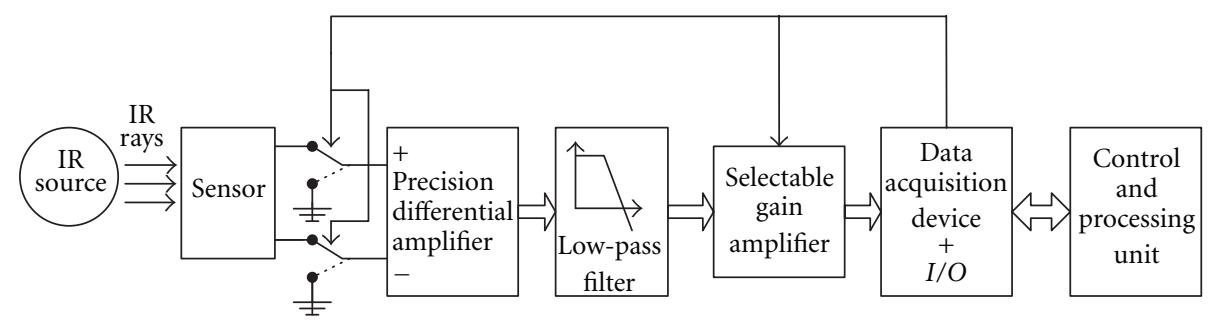

FIGURE 6: system for detecting infrared radiations.

track, covered with resin of great absorptivity (0.9), receives heat radiations emitted by the source, temperature gradients are generated between the successive junction points in accordance with the infrared radiations intensity.

\section{Experimental Results}

Infrared radiation is used in a wide variety of applications, and new applications are constantly being developed. The measurement of an infrared radiation presents a big industrial interest. In this study, the system for detecting infrared is configured as shown in Figure 6.

Infrared source includes blackbody radiators, tungsten lamps, and infrared lasers. Since the sensor output could be expressed in millivolts or even in microvolts, a special attention should be given to the measuring device. The signal conditioning circuitry (Figure 6) includes a precision differential amplifier with very low input offset voltage, and high input impedance, fully compatible with a Seebeck voltage measurement. This first amplifying stage is followed by a low-pass filter with a cutoff frequency of $100 \mathrm{~Hz}$, allowing the elimination of noise spectral components beyond that limit, but at the same time largely sufficient to leave practically unchanged the sensor's response profile. The captor's behavior to the received heat flow variations is in fact rather slow, with a time constant approaching $0.5 \mathrm{~s}$.

A further amplification is performed by a selectable gain stage to make the signal level meet the requirements for proper digitization and data acquisition.

After the ambient temperature being controlled and assessed, the power of the infrared source and the distance to the sensor set to the desired values, the measuring of the response to the incident infrared rays will be held in two stages. First, both inputs of the precision differential amplifier are switched to zero volts. The signal measured at the end of the amplifying chain is then the contribution of noise voltages, offset voltages and Seebeck voltages due to contacts between different metals at connection or soldering points.

In a second time, the inputs of the differential stage are switched to the captor's outputs. A new voltage is measured at the output of the amplifying chain. Of course, each time an analog to digital conversion and a data acquisition are executed.

Finally a personal computer that stands for a control and processing unit calculates the difference between the two

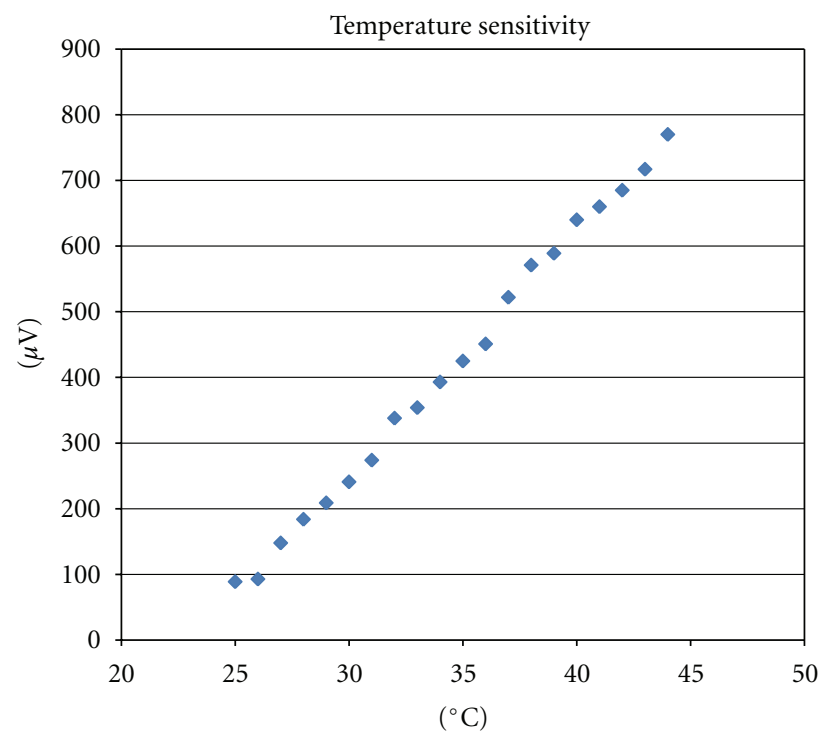

FIGURE 7: variation of the output voltage against the temperature (power source: $100 \mathrm{~W}$, distance $20 \mathrm{~cm}$ ).

measures, revealing the response of the sensor, largely freed from the signal conditioning device imperfections.

In order to reduce the impact of noise signals, including the sensor's internal noise, these measuring operations are repeated several times for each point and only the average values are taken into account.

The size of constantan track (folded up as shown in Figure 2) is length $280 \mathrm{~mm}$ and width $0.4 \mathrm{~mm}$; space between plated electrodes is $4 \mathrm{~mm}$ and the active surface of sensor is $11.8 \mathrm{~cm}^{2}$.

The experimental results enable us to trace a variation of the output voltage against the sensor's body temperature as shown in Figure 7. The calibration curve is linear and the value of the curve slope is $S=36.84 \mu \mathrm{V} /{ }^{\circ} \mathrm{C}$. Other measures are realized, when the sensor is placed at different distances from the infrared source. The output voltage against the distance for different powers radiated from a point source is shown in Figure 8.

Figure 9 shows that the output voltage is inversely proportional to the square of the distance.

These results enable us to trace the relationship between the output voltage per power received by the active surface of the sensor and the distance between power source and sensor (Figure 10).

The curves are linear and the slopes are given by Table 1 . 


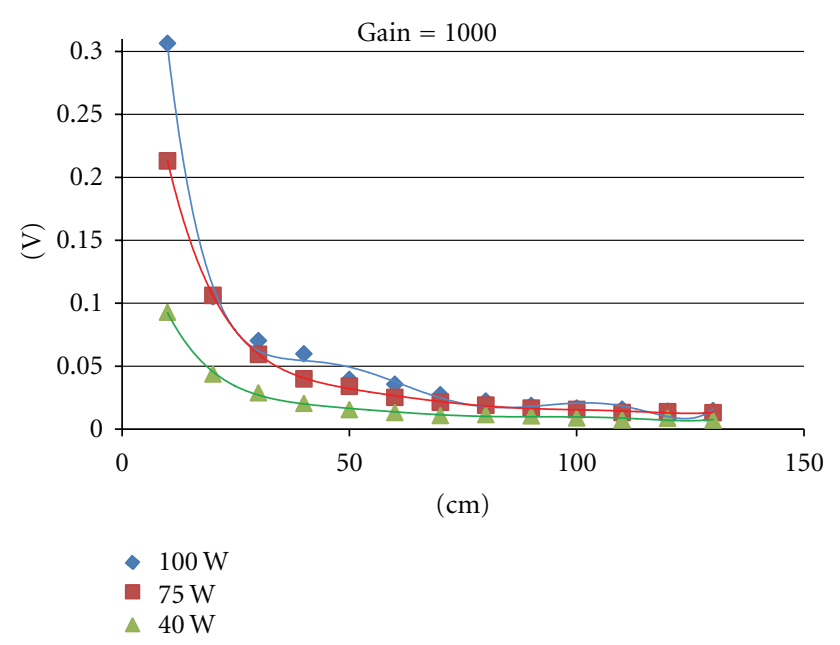

FIGURE 8: variation of the output voltage against the distance.

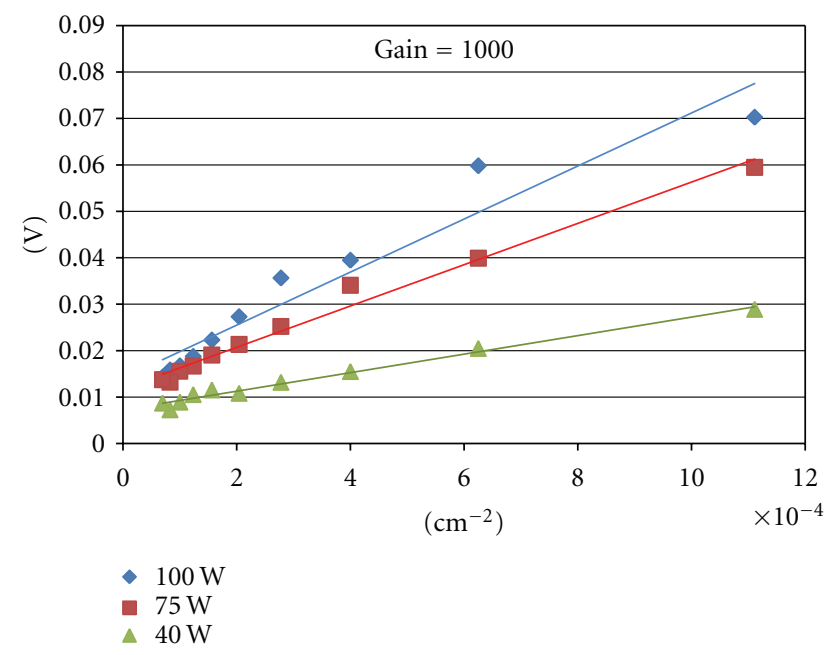

FIGURE 9: variation of the output voltage against the inverse of the square of the distance.

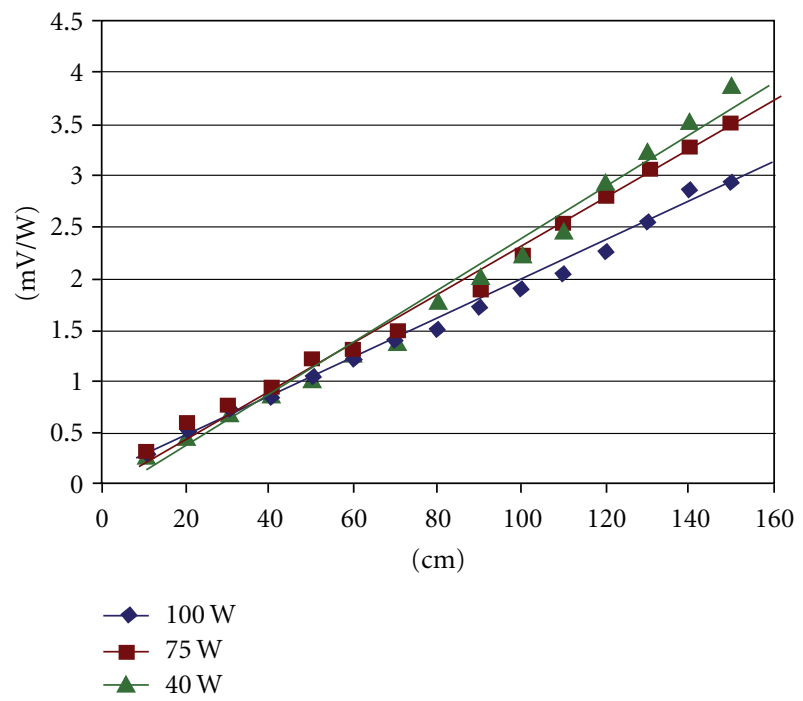

FIGURE 10: variation of the output voltage per power against the distance between source and sensor.
TABLE 1

\begin{tabular}{lccc}
\hline Source power $(\mathrm{W})$ & 100 & 75 & 40 \\
\hline Sensitivity $\left(\mu \mathrm{V} \cdot \mathrm{W}^{-1} \mathrm{~cm}^{-1}\right)$ & 19.3 & 23 & 26.7 \\
\hline
\end{tabular}

\section{Conclusion}

This study demonstrates that the constantan track placed on the top of the bimetallic circuit enabled us to realize infrared radiations detector. This sensor is sensitive and can be applied for many industrial applications. As an application, the sensor can be adapted to detect the body presence.

\section{References}

[1] R. L. Weber, Heat and Temperature, Annales de l'institut Henri Poincaré (A) Physique, prentice-Hall, Englewood Cliffs, NJ, USA, 1941, Temperature, its measurement and control in science and industry.

[2] T. Kwikketrs, "Two thick film sensors," Hybrid Circuit Technology, vol. 16, pp. 32-42, 1988.

[3] P. Herin and P. Thery, "Measurement on the thermoelectric properties of thin sensors," Measurement Science and Technology, vol. 3, pp. 495-500, 1992.

[4] P. Thery and J. Pauquet, "Propriétés thermoélectriques des systèmes à deux couches conductrices superposées," Journal of Physics E, vol. 13, pp. 323-327, 1980.

[5] D. Leclerq, F. Wattiau, and P. Thery, "Procédé et dispositif utilisant les effets thermoélectriques pour la mesure d'une grandeur physique dont la variation est apte à modifier les propriétés thermophysiques d'un milieu," French Patent 8916809.

[6] E. Gaviot, F. Polet, F. Raucoules, F. Brachelet, and K. Ziouche, "Planar differential radiometers: a quantitative approach to designing enhanced units," Measurement Science and Technology, vol. 10, no. 2, pp. 84-92, 1999.

[7] B. Bêche, L. Camberlein, F. Polet, and E. Gaviot, "A matrix formulation to introduce the concept of effective thermoelectric tensors in idealized superlattices," Sensors and Actuators Physical A, vol. 122, no. 2, pp. 209-214, 2005.

[8] K. Hachami, D. Moussaid, M. Rahmoun, and A. El Hassani, "Design and realization a new thermoelectric sensor. Application," Active and Passive Electronic Components, vol. 23, no. 3, pp. 145-155, 2000.

[9] K. Hachami, D. Moussaid, M. Rahmoun, and E. Bendada, "Utilization of the thermoelectric sensor to realize a new fluid level detector," Active and Passive Electronic Components, vol. 24, no. 3, pp. 177-186, 2001.

[10] E. Gaviot, Conception et Optimization des Radiometers à Absorption Différentielle Distribuée, Habilitation à Diriger des Recherches, Université de Lille, Lille, France, 1998. 

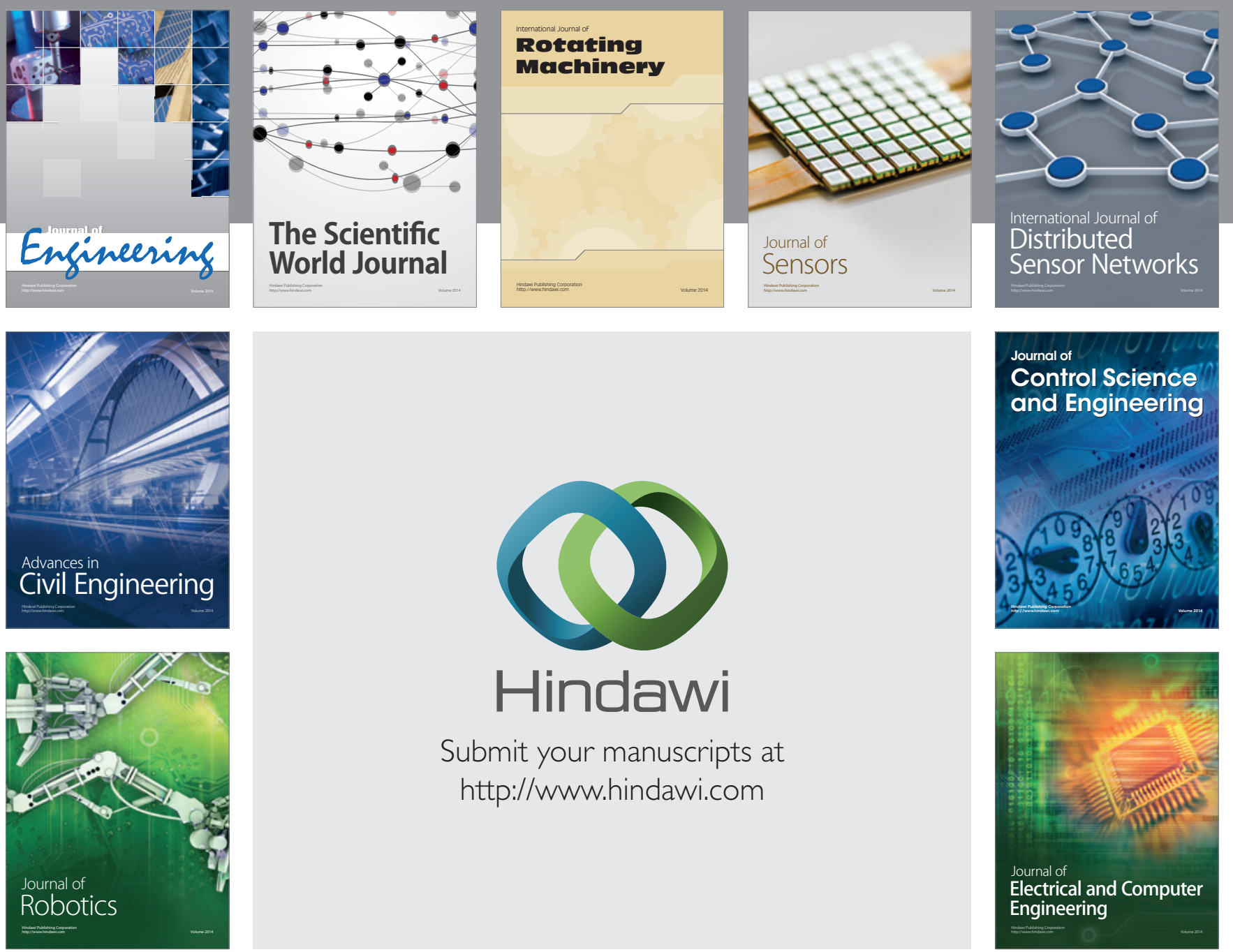

Submit your manuscripts at

http://www.hindawi.com
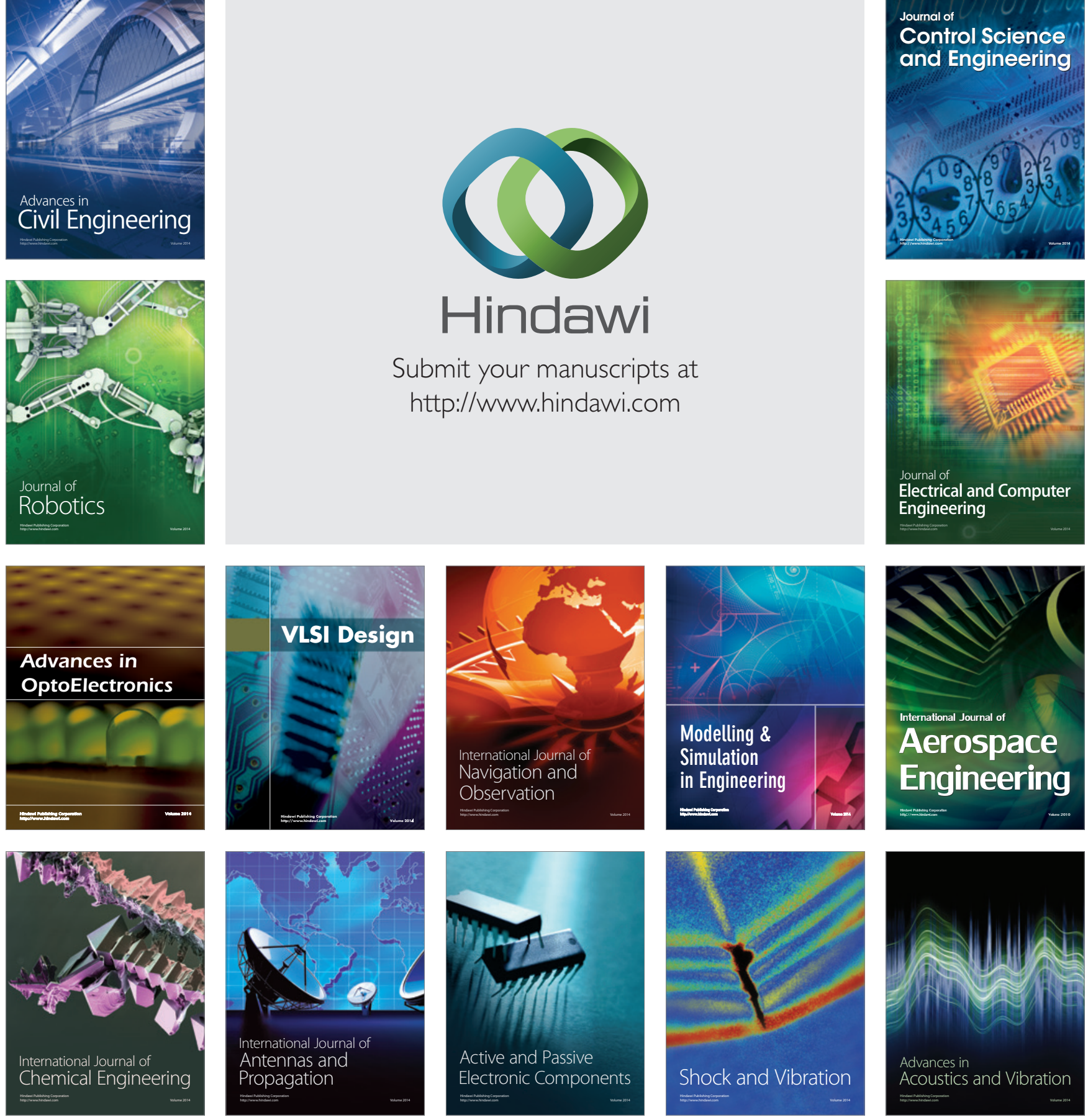